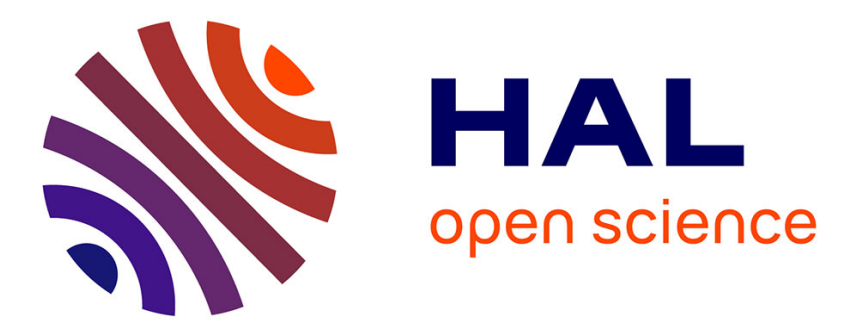

\title{
A weak-scatterer potential flow theory-based model for the hydroelastic analysis of offshore wind turbine substructures
}

Leroy Vincent, E.E. Bachynski-Polić, A. Babarit, P. Ferrant, J.-C. Gilloteaux

\section{- To cite this version:}

Leroy Vincent, E.E. Bachynski-Polić, A. Babarit, P. Ferrant, J.-C. Gilloteaux. A weak-scatterer potential flow theory-based model for the hydroelastic analysis of offshore wind turbine substructures. Ocean Engineering, 2021, 238, pp.109702. 10.1016/j.oceaneng.2021.109702 . hal-03361432

\section{HAL Id: hal-03361432 \\ https://hal.science/hal-03361432}

Submitted on 1 Oct 2021

HAL is a multi-disciplinary open access archive for the deposit and dissemination of scientific research documents, whether they are published or not. The documents may come from teaching and research institutions in France or abroad, or from public or private research centers.
L'archive ouverte pluridisciplinaire HAL, est destinée au dépôt et à la diffusion de documents scientifiques de niveau recherche, publiés ou non, émanant des établissements d'enseignement et de recherche français ou étrangers, des laboratoires publics ou privés. 


\title{
A weak-scatterer potential flow theory-based model for the hydroelastic analysis of offshore wind turbine substructures
}

\author{
V. Leroy ${ }^{a, *}$, E. E. Bachynski ${ }^{b}$, A. Babarit ${ }^{a}$, P. Ferrant ${ }^{a}$ and J.-C. Gilloteaux ${ }^{a}$ \\ ${ }^{a}$ LHEEA, Centrale Nantes/CNRS, 1 rue de la Noë 44300 Nantes, France \\ ${ }^{b}$ Department of Marine Technology, NTNU, 7491 Trondheim, Norway
}

\section{ARTICLE INFO}

\section{Keywords:}

Hydroelastic coupling

Nonlinear hydrodynamics

Weak-Scatterer approximation

Offshore wind turbine foundation

\begin{abstract}
A B S TR ACT
This paper examines the hydroelastic response of a monopile structure, supporting an offshore wind turbine. A new numerical simulation tool is presented, coupling a nonlinear potential flow solver to a structural model based on modal superposition. The hydrodynamic solver is based on the WeakScatterer (WS) approach and assumes small perturbations of the incident flow. A finite element method (FEM) solver is used to compute the modal parameters, which are then superposed to compute the dynamics of the system in time domain. Small deformations are assumed in the coupling. The two theories are tightly coupled in time domain. The theory of the coupling is fully described in the paper. The new coupled solver WS_CN-FEM is then applied to the case of a large diameter monopile. Results are compared to simulations using the Morison equation to compute the hydrodynamic loads and a beam element FEM model to compute the response of the structure and to experimental measurements made on a monopile-based offshore wind turbine model. The physical model has Froude-scaled geometry and natural frequencies, which allows an accurate validation of the hydroelastic numerical models including realistic flexible modes of the structure and wave-structure interaction. The results of WS_CN-FEM show a good agreement with the experimental measurements, and in particular on the first and second mudline bending moments' harmonics in a series of regular waves of various wave steepness.
\end{abstract}

\section{Introduction}

The size of offshore wind turbines is increasing and now reaching over $200 \mathrm{~m}$ in rotor diameter. Similarly, the size and weight of the foundations, either bottom-fixed or floating, are also increasing. Hydroelastic analysis may hence become essential to estimate internal loads in the platforms and foundations, as for any other very large structure at sea. The challenge shall be overcome for both bottom-fixed and floating wind turbines.

For hydrodynamically slender structures (e.g. jacket foundations, some monopiles or spar platforms), Morison's equation [9] can be applied. These solvers are easy to implement and can be coupled with e.g. a finite element solver for straightforward hydroelastic analysis, inducing a relatively low simulation cost. The undisturbed incident waves used in these models can include fully nonlinear wave kinematics such as the Rienecker-Fenton model for regular waves [24], or a High Order Spectral (HOS) model for irregular sea states [8]. For larger geometries, when viscous effects are negligible, potential flow theory should be used. These solvers require a mesh of the wet body surface and a hydroelastic coupled analysis is not straightforward. A one-way coupling is usually used to design large floating structures [7], considering the hydrodynamic loads computed on a rigid body and thereafter applied on a finite element mesh to compute the internal loads. The mitigation of the hydrodynamic loads induced by the structure deformation is hence not included in the analysis.

\footnotetext{
${ }^{*}$ Corresponding author

vincent.leroy@ec-nantes.fr (V. Leroy) ORCID(s):
}

For floating wind turbines, numerous numerical simulation tools have been recently developed [25], mostly coupling offshore design tools including various hydrodynamic solvers to aero-elastic solvers used to design wind turbines. They often model a flexible wind turbine (tower and rotor) supported by a rigid platform. This form of "rigid-flexible" coupling ignores the flexible modes of the platforms. The main alternatives which have been explored up to now to analyse floating wind turbines platforms flexibility use either linear potential flow theory or Morison's equation:

1. The platform can be simulated using a Boundary Element Method (BEM) and modelled as a number of interacting bodies, linked with each other using beam finite elements [13];

2. Hydrodynamic pressures computed by a BEM on a single rigid body can be projected on flexible platform sections for calculation of the deformation [22];

3 . Flexible modes can be included in the linear potential flow solver (BEM), and integrated in time domain with the servo-aero-elastic model[4];

4. Morison's equation can be used on a rigid or flexible platform formed of slender members [32].

For both bottom-fixed and floating wind turbine foundations, including nonlinear hydrodynamics can be important for the estimation of fatigue loading or ultimate design loads where waves kinematics and induced loads are very nonlinear [26]. For hydrodynamically slender structures, Morison's equation allows the inclusion of nonlinear wave kinematics. For example, compared to simulations using linear wave kinematics, simulations of a semi-submersible with 
nonlinear wave kinematics resulted in approximately $25 \%$ larger extreme mooring line tensions [32].

For increasing structure diameter, however, near-field diffraction, which is not captured by Morison's equation, become more significant. Although first-order solutions such as the MacCamy-Fuchs equation exist, combining near-field diffraction effects with nonlinear waves remains challenging.

High-order diffraction loads acting on bottom-fixed cylindrical foundations can also be computed analytically using Kristiansen and Faltinsen's (KF) extension of the FNV model $[10,17]$ but it is applicable to simple geometries, and assumes long waves relative to the structure. The KF model has been shown to overestimate the third order forces for steep waves [17]. Both the KF model and Rainey's model tend to overpredict the first mode response of a fully flexible monopile in irregular waves compared to experimental results [29]. Fully non-linear potential flow models have also been developed $[11,12]$ but they suffer from important numerical instabilities. Alternatively, weakly non-linear potential flow models, such as the weak-scatterer approach have been used to compute the ringing response of a bottomhinged, rigid monopile structure in focused waves [33].

Nonlinear coupled models have been developed for ships, including forward speed [15, 19], showing good performance. This paper presents a similar model that has been developed for offshore floating wind turbines: WS_CN-FEM. A weakly nonlinear potential flow solver based on the Weakscatterer theory, WS_CN, has been developed in Centrale Nantes [21, 6, 31]. It has been recently coupled to a low order finite element solver, based on Euler-Bernoulli beam model which assumes small deformations of slender beams. The developed solver is first tested and validated on the case of bottom-fixed wind turbines in this paper before being tested for floating structures in future works.

The paper presents the theory behind the non-linear hydroelastic coupling WS_CN-FEM: it presents the weak-scatterer approach, the structural model and the tight coupling between the two through an explicit coupled equation system. Coupled simulations of a representative monopile supported offshore wind turbine are then performed and compared to experimental measurements performed on a scaled large-diameter flexible monopile [2] in regular waves. Simulations from SIMA [27], using Morison's equation and nonlinear wave kinematics, are also presented in the comparison.

\section{Hydroelastic weak-scatterer theory based solver}

The nonlinear potential flow solver solver WS_CN has been developed at Centrale Nantes since 2011. It initially was developed to model underwater wave energy converters [21], and it has been lately improved to account for freesurface piercing [6] and interacting bodies [31]. WS_CN has now been coupled to a structural solver to solve the hydroelastic coupling on offshore wind turbines substructures. The new coupling is called WS_CN-FEM.

\subsection{Structure model}

The Euler-Bernoulli beam theory, which assumes small deformations, has been implemented in a new solver called Beampy. The finite elements are linearized, and the stiffness and mass matrices $\mathbf{K}$ and $\mathbf{M}$ of the system are computed at the initial position and kept constant throughout the simulations.

The hydrodynamic solver requires an explicit scheme for solving the equation of motion, which necessitates time steps shorter than the shortest natural period in the structure model. In order to lower the CPU cost of the simulations, the equation of motion is therefore solved using modal superposition. A sufficient number of modes needs to be considered for an accurate modelling of the dynamics, and high order modes are ignored in order to lengthen the integration time step while keeping the simulations stable. A preliminary convergence study is hence performed to define the needed number of modes in the analysis.

Before simulating the response of the structure in timedomain, a modal analysis is performed. If the system is floating, a linear stiffness corresponding to hydrostatic and mooring stiffness is added to constrain all rigid degrees of freedom. In this modal analysis, hydrodynamic added mass is ignored. The modal matrix $\psi$ has the shape $\left(n_{\text {nodes }} \times n_{\text {modes }}\right)$ and gives the structural mode shapes for the considered system.

Stiffness-proportional Rayleigh damping is applied in the damping matrix $\mathbf{C}$. In time domain, the equation of motion takes the form given in Equation (1), where $\boldsymbol{y}$ is the modal response amplitude, $\mathbf{F}^{H}$ the vector of hydrodynamic forces and $\mathbf{F}^{\text {ext }}$ the vector of other external forces.

$$
\psi^{T} \mathbf{M} \psi \ddot{y}+\psi^{T} \mathbf{C} \psi \dot{y}+\psi^{T} \mathbf{K} \psi y=\psi^{T}\left(\mathbf{F}^{H}+\mathbf{F}^{e x t}\right)
$$

where the position vector $\boldsymbol{x}$ is obtained from the amplitudes of the modal responses $y$ as written in Equation (2).

$$
x=\psi y
$$

The procedure used for simulations with $W S \_C N-F E M$ is described in Figure 1.

The hydrodynamic added mass, not included in the preliminary modal analysis, is then included in the hydrodynamic force calculated on the body. This is detailed in Section 2.3.

\subsection{Flow model}

Let us consider a body undergoing motions in a fluid domain. Its motions are decomposed into rigid and flexible motions, i.e. structural deformations. In this fluid-structure coupling, the fluid problem is linearized around the surface of the rigid body at its instantaneous position, disregarding structural deformation. The instantaneous position is defined by the superposition of the rigid modes, neglecting the flexible modes of the structure presented in Section 2.1. The cylindrical fluid domain $\mathcal{D}$ is presented in Figure 2. It is delimited by the side numerical tank walls $S_{W}$, the seabed $S_{D}$, 
A WS theory-based model for the hydroelastic analysis of OWT substructures

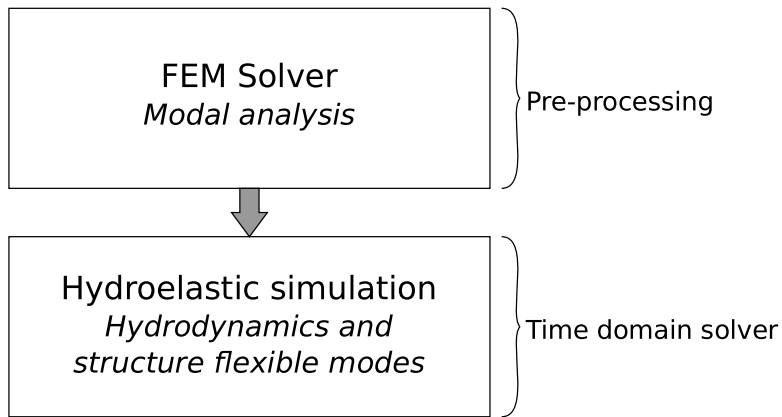

Figure 1: Simulation procedure with WS_CN-FEM. Flexible modes are computed during the pre-processing and used later in the coupled time-domain simulation with the hydrodynamic solver.

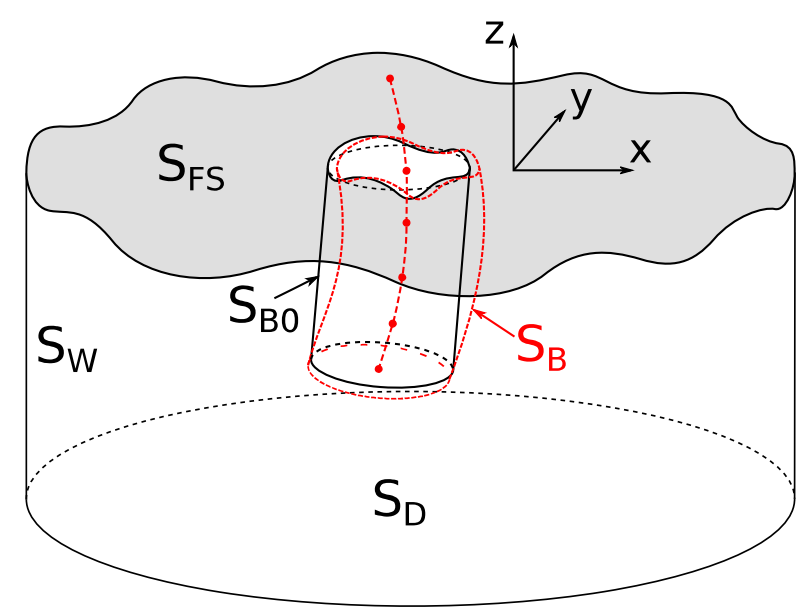

Figure 2: The cylindrical fluid domain $\mathcal{D}$ considered in the WS_CN solver. The fluid domain $\mathcal{D}_{0}$ is delimited by $S_{W}, S_{D}$, $S_{F S}$ and the wet surface of the rigid body $S_{B 0}$.

the free-surface $S_{F S}$ and the wet body surface $S_{B}$. The fluid domain $\mathcal{D}_{0}$ is then delimited by $S_{W}, S_{D}, S_{F S}$ and the wet surface of the rigid body $S_{B 0}$. For a bottom-fixed foundation, $S_{B 0}$ corresponds to the surface of the body at rest.

The Euler-Bernoulli theory assumes small linear deformations, hence the wet surface of the body equals the wet surface of the associated rigid body.

$$
S_{B} \approx S_{B 0}
$$

The weak-scatterer theory assumes an inviscid, incompressible and irrotational flow. The Laplace equation is then written for the velocity potential.

$$
\Delta \phi=0
$$

The velocity potential $\phi$ and the free-surface elevation $\eta$ are decomposed into an incident (undisturbed) and a perturbed (or scattered) quantity. The weak-scatterer approach assumes that the perturbed quantity is small compared to the incident wave, as in Equation (5). The incident quantities can be computed using either Airy linear wave theory, or fully non-linear Rienecker-Fenton wave theory [24].

$$
\left\{\begin{array} { l } 
{ \phi = \phi ^ { I } + \phi ^ { P } } \\
{ \eta = \eta ^ { I } + \eta ^ { P } }
\end{array} \quad \text { with } \quad \left\{\begin{array}{l}
\phi^{P}=o\left(\phi^{I}\right) \\
\eta^{P}=o\left(\eta^{I}\right)
\end{array}\right.\right.
$$

The far field condition (6) writes that the perturbed quantities are negligible when far from the body.

$$
\left\{\begin{array}{l}
\phi^{P} \underset{r \rightarrow+\infty}{\longrightarrow} 0 \\
\eta^{P} \underset{r \rightarrow+\infty}{\stackrel{\longrightarrow}{\longrightarrow}} 0
\end{array}\right.
$$

The kinematic and dynamic free-surface boundary conditions are written at the incident and undisturbed free-surface elevation $\eta^{I}$, using a Taylor expansion [21]. The wet body surface is defined as the surface of the body below $\eta^{I}$. The exact intersection of the body mesh with the undisturbed free-surface is hence calculated at each time step in the solver.

The slip boundary condition of the velocity potential is written on $S_{B 0}$, accounting for the velocities induced by the deformations. On the other domain boundaries, a slip condition is written for the perturbed potential $\phi^{P}$ on the side walls $S_{W}$ and on the full potential $\phi$ for the seabed $S_{D}$. A numerical absorbing beach is used on the outer area of the free-surface $S_{F S}$ to avoid any reflection of the scattered wave. The boundary conditions on the potential are written in Equation (7).

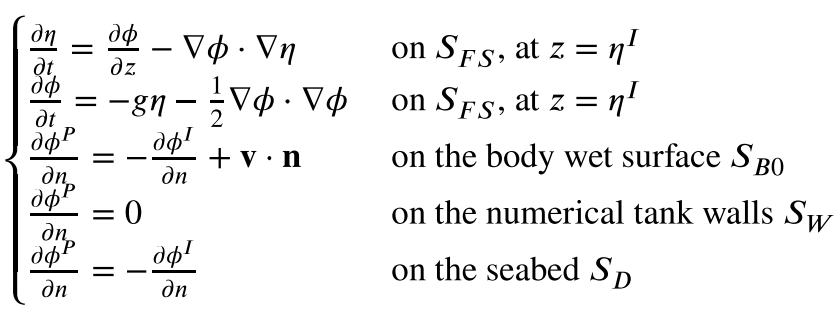

$\mathbf{v}$ and $\mathbf{n}$ are respectively the velocity of a wet body mesh node and the normal vector at this node, on $S_{B 0}$, oriented outward from the body. All terms of order $O\left(\left(\phi^{P}\right)^{2}\right)$, $O\left(\left(\eta^{P}\right)^{2}\right)$ and $O\left(\phi^{P} \times \eta^{P}\right)$ are ignored.

The second Green identity is written for the linearized fluid domain $\mathcal{D}_{0}$ on its boundary $S_{0}$ for the perturbation potential $\phi^{P}$, that satisfies the Laplace equation, and the Rankine source Green's function $G$. At all points $M_{0}$ of $S_{0}$, it takes the form given in Equation (8).

$$
\begin{aligned}
\Omega(M) \phi^{P}(M) & +\iint_{S_{0}} \phi^{P}(M) \frac{\partial G}{\partial n}(M, P) d S \\
& -\iint_{S_{0}} G(M, P) \frac{\partial \phi^{P}}{\partial n}(M) d S=0
\end{aligned}
$$


$\Omega(M)$ is explicitly written in Equation (9). It can be interpreted as the solid angle of the surface $S$ seen from the point $M$.

$$
\Omega(M)=-\iint_{S_{0}} \frac{\partial G}{\partial n}(M, P) d S
$$

The fluid domain boundaries are discretized with triangular panels [31], except for the flat seabed on which a symmetry condition is applied. The first boundary value problem (BVP1) in Equation (8) can be written as a linear system, written in a matrix format in Equation (10).

$$
\mathbf{G} \boldsymbol{\phi}^{P}=\mathbf{H} \boldsymbol{\phi}_{, n}^{P}
$$

The vectors $\phi$ and $\phi_{, n}^{P}$ respectively take the discrete values of $\phi^{P}$ and $\phi_{, n}^{P}=\frac{\partial \phi^{P}}{\partial n}$ on the domain mesh nodes. $\mathbf{G}$ and $\mathbf{H}$ are called influence coefficients matrices and represent the influence of every node on every other node of the domain mesh.

This linear system of equations is solved to compute the flow state, using a generalized minimal residual (GMRES) solver. The calculation of the hydrodynamic loads implies a fluid structure coupling detailed in Section 2.3.

The first developments of this hydrodynamic solver have been validated on rigid fixed, and floating bodies, immersed and surface-piercing [20, 6, 31]. The following theory has been developed and implemented to extend the capabilities of the solver to flexible bodies with small deformations.

\subsection{Fluid-structure coupling}

The hydrodynamic force vector is calculated by integration of the hydrodynamic pressure on the linearized wet surface of the hull $S_{B}$.

$$
\mathbf{F}^{H}=-\iint_{S_{B 0}} p^{H} \boldsymbol{n d} S
$$

$p^{H}$ is the hydrodynamic pressure computed by the WS_CN solver. According to the Bernoulli-Lagrange formula and to the Weak-Scatterer hypothesis given in Equation (5), $p^{H}$ shall be calculated as written in Equation (12).

$$
p^{H}=-\rho\left(\frac{\partial \phi^{I}}{\partial t}+\frac{\partial \phi^{P}}{\partial t}+\frac{1}{2} \nabla \phi^{I} \cdot \nabla \phi^{I}+\nabla \phi^{I} \cdot \nabla \phi^{P}+g z\right)
$$

In Equation (12), the term $\frac{\partial \phi^{P}}{\partial t}$ is unknown. The hydrodynamic force is then decomposed as in Equation (13), where $\mathbf{F}_{0}^{H}$ is the integration of the known part of the pressure.

$$
\mathbf{F}^{H}=\mathbf{F}_{0}^{H}+\rho \iint_{S_{B 0}} \frac{\partial \phi^{P}}{\partial t} \mathbf{n} d S
$$

Solving the equation of motion (1) hence involves calculating both the modal acceleration $\ddot{\mathbf{y}}$ and the derivative of the scattered velocity potential with respect to time $\left(\frac{\partial \phi^{P}}{\partial t}\right)$ at the same time.

This coupled problem could be solved using an iteration process between the structure and fluid domains, which would induce a very high CPU cost. Instead, an implicit boundary method is used [21], writing a second boundary value problem to compute $\frac{\partial \phi^{P}}{\partial t}$. The second Green identity links $\frac{\partial \phi^{P}}{\partial t}$ to its normal gradient $\frac{\partial^{2} \phi^{P}}{\partial n \partial t}$ on the wet hull surface, similarly to Equation (8).

$$
\begin{aligned}
\Omega(M) \frac{\partial \phi_{p}(M)}{\partial t} & -\iint_{S_{0}} \frac{\partial \phi_{p}(P)}{\partial t} \frac{\partial G(M, P)}{\partial n} d S \\
& +\iint_{S_{0}} \frac{\partial^{2} \phi_{p}(P)}{\partial n \partial t} G(M, P) d S=0
\end{aligned}
$$

This problem is solved using the boundary conditions given in Equation (15) which are derived from the boundary conditions of the first problem given in Equation (7). The conditions on the free-surface are not detailed here.

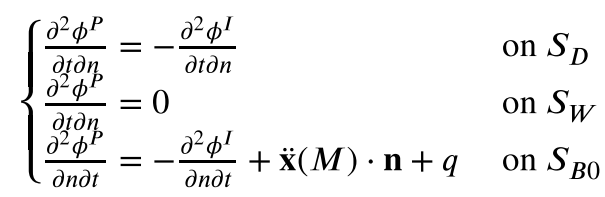

$\ddot{\mathbf{x}}(M)$ is the acceleration of a point $M$ on the body wet surface, calculated from $\ddot{\mathbf{y}}$ and $q$ is a convection term that is calculated analytically [21].

The second boundary value problem (14), the boundary condition on the wet surface (15) and the equation of motion (1) are written in a single linear system given in Equation (16). It is solved at each time step using a GMRES solver to compute $\frac{\partial \phi^{P}}{\partial t}$ and $\ddot{\mathbf{y}}$.

$$
\left\{\begin{array}{l}
\mathbf{G} \dot{\phi}^{P}=\mathbf{H} \dot{\phi}_{n}^{P} \\
\boldsymbol{\psi}^{T} \mathbf{M} \psi \ddot{\mathbf{y}}-\boldsymbol{\psi}^{T} \mathbf{L} \dot{\boldsymbol{\phi}}^{P}\left(S_{B}\right)=-\boldsymbol{\psi}^{T} \mathbf{C} \boldsymbol{\mathbf { y }}-\psi^{T} \mathbf{K} \psi \mathbf{y} \\
\quad+\boldsymbol{\psi}^{T}\left(\mathbf{F}_{0}^{W S C}+\mathbf{F}^{e x t}\right) \\
\dot{\phi}_{n}^{P}\left(S_{B 0}\right)-\mathbf{D} \psi \ddot{\mathbf{y}}=-\dot{\boldsymbol{\phi}}_{n}^{I}\left(S_{B 0}\right)+\mathbf{B}+\mathbf{Q}
\end{array}\right.
$$

The influence coefficients matrices $\mathbf{G}$ and $\mathbf{H}$ are the same as in the first boundary value problem given in Equation (10). The matrix $\mathbf{L}$ represents the integration of the time derivative of the scattered velocity potential $\dot{\phi}^{P}$ over $S_{B 0}$. $\mathbf{L}$ is calculated based on the geometry of the body mesh only. The matrix $\mathbf{D}$ and the vector $\mathbf{B}$ enable calculation of the mesh nodes' accelerations as a function of the accelerations of the structural mesh nodes. $\mathbf{D}$ and $\mathbf{B}$ are calculated as functions of the geometry of the body mesh and its velocity, known at this step of the calculation. The matrices $\mathbf{L}$ and $\mathbf{D}$ and 


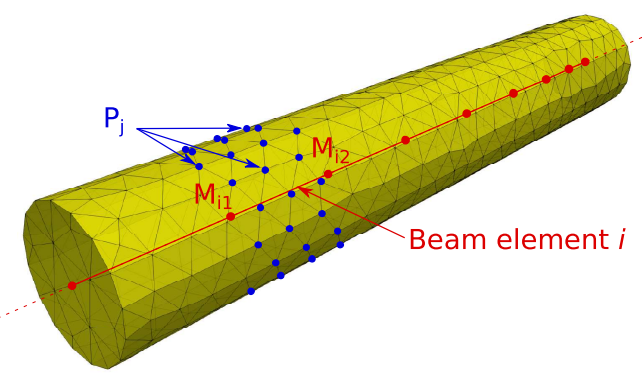

Figure 3: Projections of the structure and hydrodynamic mesh.

vector $\mathbf{B}$ are computed based on the projection of the hydrodynamic and structural mesh on one another, using standard Euler-Bernoulli beam theory shape functions. Figure 3 represents the hydrodynamic mesh nodes $P_{j}$ corresponding to one beam element defined between the nodes $M_{i 1}$ and $M_{i 2}$. The coefficients of $\mathbf{L}, \mathbf{D}$ and $\mathbf{B}$ are computed analytically. The vector $\mathbf{Q}$ contains the discrete convection terms $q$ seen in Equation (15).

This coupling is solved in the time domain and integrated using a $4^{\text {th }}$ order Runge-Kutta (RK4) explicit integration.

As introduced in section 2.1, the structure response needs to be represented by a sufficient number of modes, generally imposing a smaller time-step than that of the hydrodynamic solver. The objective is then to keep a good compromise between CPU cost and accuracy on the hydrodynamic time step, with a smaller time-step for the structural dynamics. The chosen solution involves a secondary integration scheme for the structure to stably advance its dynamics in time. This second RK4 scheme is synchronised with the primary RK4 scheme used to integrate the coupled system of equations (16). Starting from the coupled solution at a given time-step, it integrates the equation of motion of the structure using the computed hydrodynamic loads as a constant force, until the next synchronous time-step where the system (16) is solved. This hybrid integration scheme has been verified and validated. A demonstration is presented in Appendix A.

\section{Verification and validation}

\subsection{Case study and experiments}

The study considers the DTU $10 \mathrm{MW}$ wind turbine [3] on a $9 \mathrm{~m}$ diameter monopile foundation [30] subjected to different regular wave systems. The WS_CN-FEM coupling is compared to both a beam element finite element solver combined with Morison's equation, implemented in SIMA [27], and experimental measurements made on an offshore wind turbine model, in hydroelastic similarity. The numerical models of the studied wind turbine are based on a model-of-themodel approach, scaled-up to full scaled, in order to optimise the comparison with the experiments.

The aim of this case study is not to prove a better accuracy with a potential flow solver than with a Morison-based solver, but to validate the theory presented in Section 2 on the case of a bottom-fixed structure, before an application to offshore floating wind turbines.

Wind-induced loads and current-induced loads are not considered in the analysis.

\subsubsection{Experimental set-up}

The experiments were performed by SINTEF Ocean and NTNU in the small towing tank of NTNU in Trondheim, with a Froude scaling factor of 50 [2], as part of the WAS$X L$ project [28]. Figure 4 presents the layout of the tank. The waves are generated with a piston-type wavemaker. Due to the short size of the tank, the experiments could not completely avoid wake reflexion on the beach and dampers, hence a wave reflection of approximately $4 \%$ is expected to be present in most presented results [16].

In the following, all quantities are given at full scale. The monopile model is mounted on a rigid foundation below the seabed. The wind turbine and the monopile are formed of a flexible backbone, providing modal frequency similarity surrounded by a cylinder respecting Froude similarity. The water depth is $27 \mathrm{~m}$ and the tower top reaches $144.15 \mathrm{~m}$ above the seabed. The total mass of the model without the Rotor-Nacelle Assembly (RNA) is $2.652 \cdot 10^{6} \mathrm{~kg}$ and its center of gravity is at $34.25 \mathrm{~m}$ above the seabed. A mass of $9.55 \cdot 10^{5} \mathrm{~kg}$ representing the RNA is added at the tower top in order to obtain a good agreement on the $1^{\text {st }}$ fore-aft bending mode frequency with the base case wind turbine. Figure 5 represents the wind turbine and foundation model. The model is equipped with strain gauges and accelerometers at several positions. We use the mudline bending moment, calculated using strain gauges, and the accelerations measured at the top of the RNA (at $145.4 \mathrm{~m}$ above the seabed), as reference measurements to validate the models.

The experimental set-up is described by Bachynski et al. [2].

The two first bending modes in the XOZ plane have periods of $T_{1}=3.97 \mathrm{~s}$ and $T_{2}=0.656 \mathrm{~s}$ respectively. The damping in the numerical models is calibrated based on experimental decay tests.

\subsubsection{Nonlinear hydroelastic model}

The developed nonlinear hydroelastic coupling $W S \_C N$ FEM is used to analyse the considered case study. As presented previously, the boundaries of the fluid domain are meshed, including the free-surface and the outer boundaries. A convergence analysis has been performed in the first place, on both the time-step length and on the mesh dimensions: domain diameter and cells' size.

The convergence analyses is not presented in this paper, however the calculation parameters are given as follows:

1. The time step used in the hydrodynamic solver is $0.1 \mathrm{~s}$, and the time-step in the structural solver is $5 \cdot 10^{-4} \mathrm{~s}$.

2. The fluid domain has a diameter equal to 3 wavelengths, with an absorption beach of one wavelength on the outer boundaries.

3. The mesh cell size is $0.7 \mathrm{~m}$ on the body boundaries and approximately $10 \%$ of the wavelength on the outer 


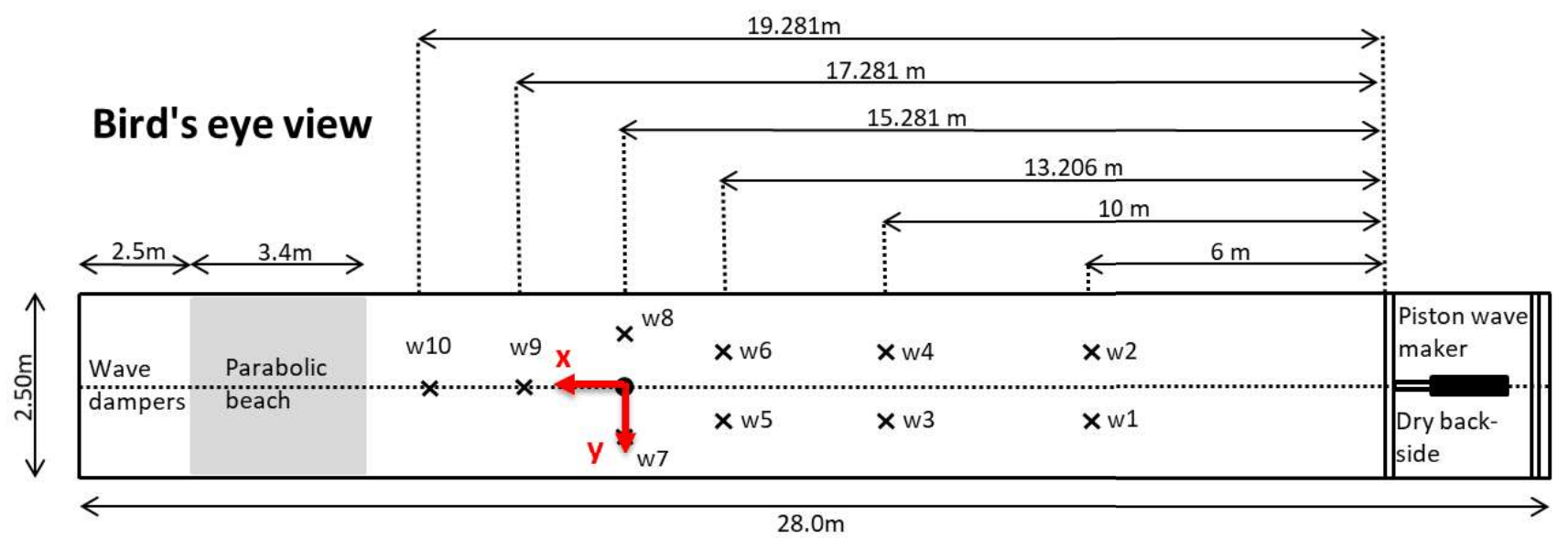

Side view

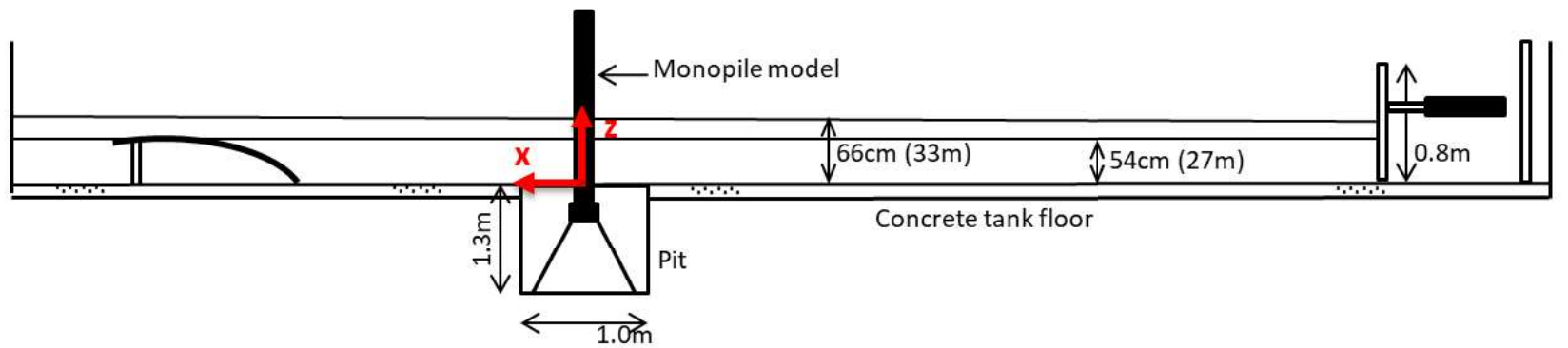

Figure 4: Layout of the experiments in the small towing tank of NTNU [2]. 10 wave gauges are used.

boundary.

The fluid domain mesh for a wave period of $6 \mathrm{~s}$, with 4600 nodes, is presented in Figure 6. A smoothing algorithm is applied every 5 time steps to the solution of the potential on the free-surface in order to avoid numerical instability.

The input waves are computed using the fully non-linear Rienecker-Fenton wave model [24].

\subsubsection{Morison-FEM model}

$W S \_C N-F E M$ is first compared to the numerical simulation tool SIMA [27], which for this study uses finite elements to model the structure response and a Morison-based hydrodynamic solver to compute the hydrodynamic loads. For the sake of comparison against WS_CN-FEM, SIMA computes the loads with a drag coefficient $C_{D}=0$ and the added-mass coefficient $C_{A}$ is defined according to the analytical solution to the linear potential flow theory for a diffracting cylinder from MacCamy and Fuchs [23] for each regular wave. The loads are applied to the beam model (Euler-Bernoulli), using Stokes $2^{\text {nd }}$ order wave kinematics. The hydrodynamic loads are integrated up to the incident $2^{\text {nd }}$ order wave elevation level and the velocity potential is extrapolated above the mean sea level $(z=0)$ using a linear extrapolation.

\subsubsection{Load cases}

First, decay tests are performed on the dry monopile and on the wet monopile, in the wave tank. A series of regular waves are then tested in the wave tank, including several wave periods, $T=6 s, 8 s$ and $10 s$, with various steepness, defined by the ratio between wave height and wavelength following $\frac{H}{\lambda}=\frac{1}{40}, \frac{1}{30}$ and $\frac{1}{22}$.

The considered regular waves are presented in Table 1 . The regular waves are also plotted in blue in Figure 7, where the validity of wave force models is presented [5] as a function of:

1. the $k R$ number such as $k R=\frac{\pi D}{\lambda}$, where $D$ is the cylinder diameter and $\lambda$ the wavelength;

2. the wave height to cylinder diameter ratio $\frac{H}{D}$, ratio similar to the Keulegan-Carpenter number.

The diffraction region (bottom right-hand corner of the Figure 7 mostly refers to the linear potential flow theory. As one can see, most regular waves are outside the diffraction region, which is expected for a monopile foundation.

The added mass coefficient used in SIMA, calculated based on the MacCamy and Fuchs solution, is also given in Table 1 for each load case.

\subsection{Modal analysis and decay tests}

Decay tests were performed on the model outside and in the water, and the corresponding acceleration measurements 
A WS theory-based model for the hydroelastic analysis of OWT substructures
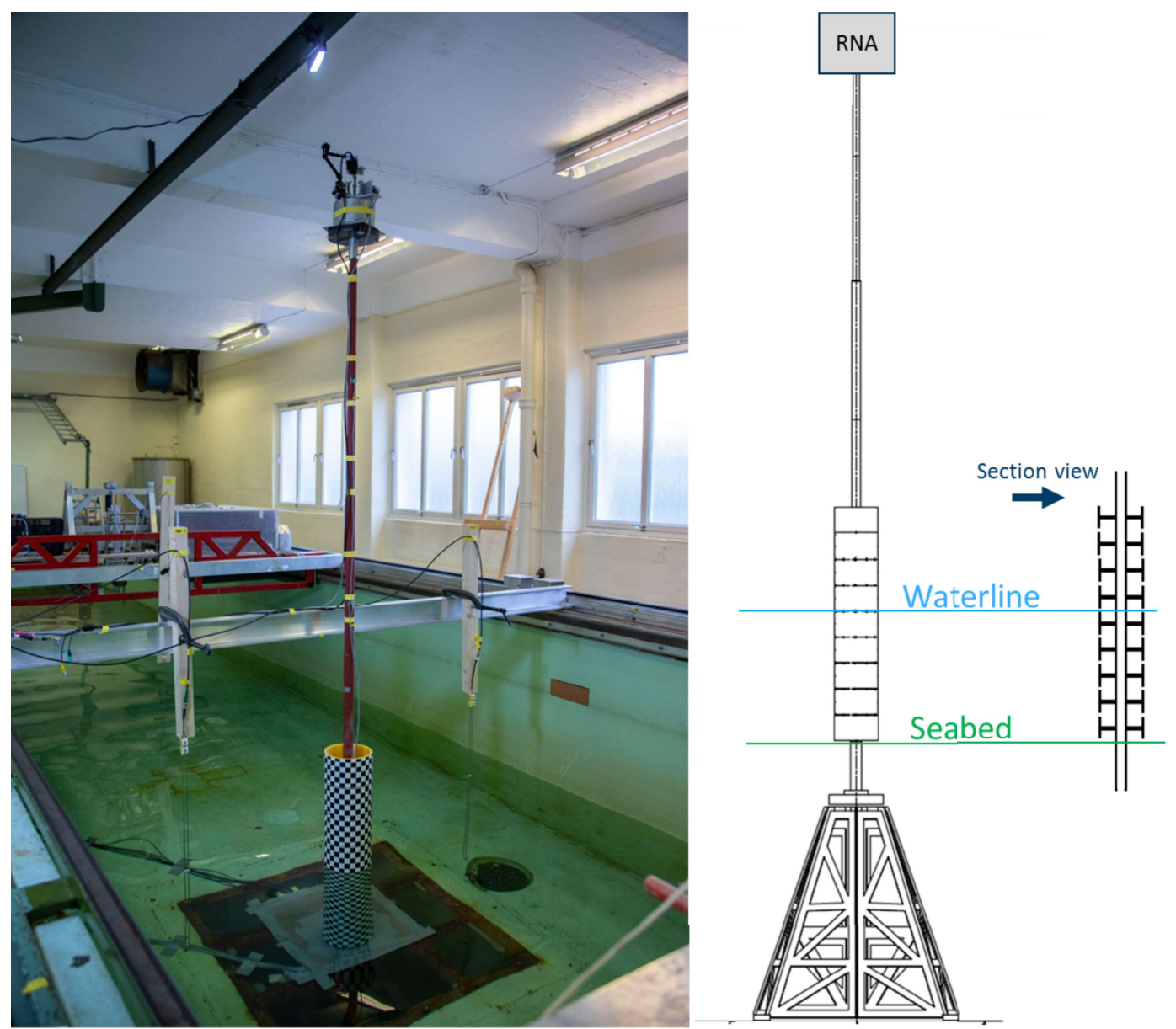

Figure 5: Wind turbine and monopile foundation model [2].

Table 1

Regular wave cases.

\begin{tabular}{cccccc}
\hline Number & Period $(\mathrm{s})$ & Height $(\mathrm{m})$ & Target H/Lambda (\%) & Measured H/Lambda $(\%)$ & $C_{A}(-)$ \\
\hline 1 & 6.000 & 1.336 & $2.500(1 / 40)$ & 2.376 & 1.0643 \\
2 & 6.000 & 1.690 & $3.333(1 / 30)$ & 2.995 & 1.0643 \\
3 & 6.000 & 2.110 & $4.545(1 / 20)$ & 3.721 & 1.0643 \\
4 & 8.000 & 2.297 & $2.500(1 / 40)$ & 2.416 & 1.0466 \\
5 & 8.000 & 3.060 & $3.333(1 / 30)$ & 3.205 & 1.0466 \\
6 & 8.000 & 4.102 & $4.545(1 / 22)$ & 4.265 & 1.0466 \\
7 & 10.000 & 3.237 & $2.500(1 / 40)$ & 2.411 & 1.03 \\
8 & 10.000 & 4.404 & $3.333(1 / 30)$ & 3.264 & 1.03 \\
9 & 10.000 & 6.002 & $4.545(1 / 22)$ & 4.405 & 1.03 \\
\hline
\end{tabular}

were used to identify the natural frequencies, mode shapes, and damping. The $1^{\text {st }}$ and $2^{\text {nd }}$ fore-aft bending mode frequencies and shapes are compared with the eigenvalue analysis from the numerical models. As shown in Figure 1, in the WS_CN-FEM solver, modal analysis is only carried out for the dry structure. For the wet case, a time-domain decay test simulation is performed using the non-linear coupling previously presented. The wet natural frequencies are then found from the decay simulation.

The mudline bending moment time series is presented in Figure 8, comparing the measurements with the simula- tions from WS_CN-FEM and SIMA. A difference in the first mode period between $W S \_C N-F E M$ and the experiments is observed. The amplitude of the response shows a very good agreement between simulations and experiments.

The observed and computed modes frequencies are given in Table 2. The normalised shapes of the two first fore-aft bending modes are plotted in Figure 9. The agreement between measurements and models is very satisfactory. The $1^{\text {st }}$ and $2^{\text {nt }}$ mode periods computed by WS_CN-FEM are slightly smaller than the measured ones, with respectively $2.9 \%$ and $0.45 \%$ relative difference. 
Table 2

Natural frequencies on the $1^{\text {st }}$ and $2^{\text {nd }}$ fore-aft modes for the dry and wet model.

\begin{tabular}{lccc}
\hline & & Dry mode period (s) & Wet mode period (s) \\
\hline \multirow{3}{*}{ Mode 1 } & WS_CN-FEM & 3.854 & 3.850 \\
& SIMA & 3.918 & 3.923 \\
& Exp. & 3.970 & 3.967 \\
\hline \multirow{2}{*}{ Mode 2 } & WS_CN-FEM & 0.584 & 0.617 \\
& SIMA & 0.587 & 0.637 \\
& Exp. & 0.610 & 0.646 \\
\hline
\end{tabular}

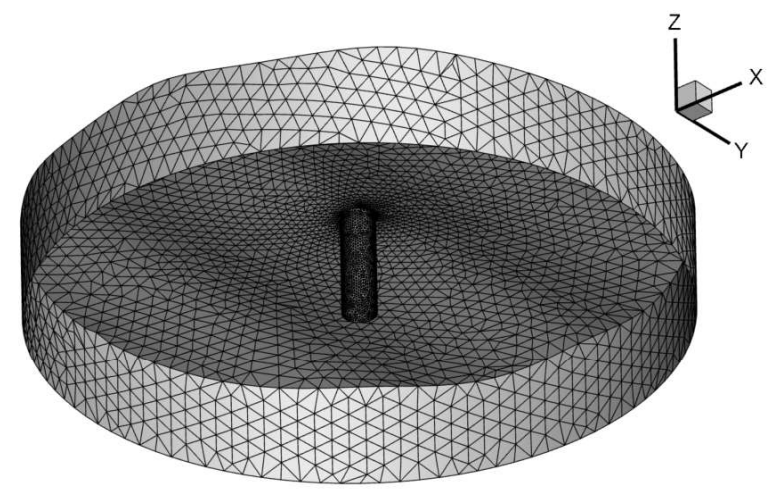

Figure 6: Fluid domain mesh, seen from below.



Figure 7: Regions of application of wave-force models for a vertical cylinder [5]

\subsection{Regular waves}

The model is studied in the regular wave conditions presented in Section 3.1.4. The input waves in the numerical simulations are based on experimental measurements without the model, using a wave probe located at the model's position (w11, not represented in Figure 4). The free surface elevation is plotted in Figure 10 for the three investigated values of wave steepness $(H / \lambda=1 / 40,1 / 30$ and $1 / 22)$ and for



Figure 8: Bending decay test, in the water.

the wave periods $\mathrm{T}=6,8$ and $10 \mathrm{~s}$. The curves represent the free-surface elevation in WS_CN-FEM, SIMA and the measurements at the model's position, without the model, and just next to the model, at w8 in Figure 4, with the model in place. There is very good agreement between the undisturbed measured wave and the simulations. The difference between the measurement at w8 and the empty tank measurement at w11 is mainly due to the wave diffraction on the cylinder. It is particularly visible in Figure $10 \mathrm{a}$ when $\mathrm{T}=6 \mathrm{~s}$ and $H / \lambda=1 / 22$.

The computed and measured mudline bending moments are plotted in Figure 11 for each wave. At $\mathrm{T}=6 \mathrm{~s}$ (Figure 11a), the $k R$ number is 0.5 and the Keulegan-Carpenter number $(\mathrm{KC})$ is sufficiently low so that the wave conditions are appropriate for application of the potential flow theory (see Figure 7). The agreement is very good except at $H / \lambda=1 / 22$, where the two simulation tools tend to underestimate the amplitude of the response compared to the experiments. This is discussed further with the harmonic analysis.

The mudline bending moment obtained in the $T=8 \mathrm{~s}$ period regular wave is plotted in Figure 11b. The response is large and it contains an important double frequency oscillation compared to responses at other wave periods. The $2^{\text {nd }}$ order hydrodynamic loads excite the first fore-aft bending mode of the structure, given in Table 2 at a period of $3.9 \mathrm{~s}$. The accuracy on this case is poorer. The response at the resonance period is very sensitive to the structural damping. Hence, any uncertainty in the structural damping may induce large errors in the coupled models. 


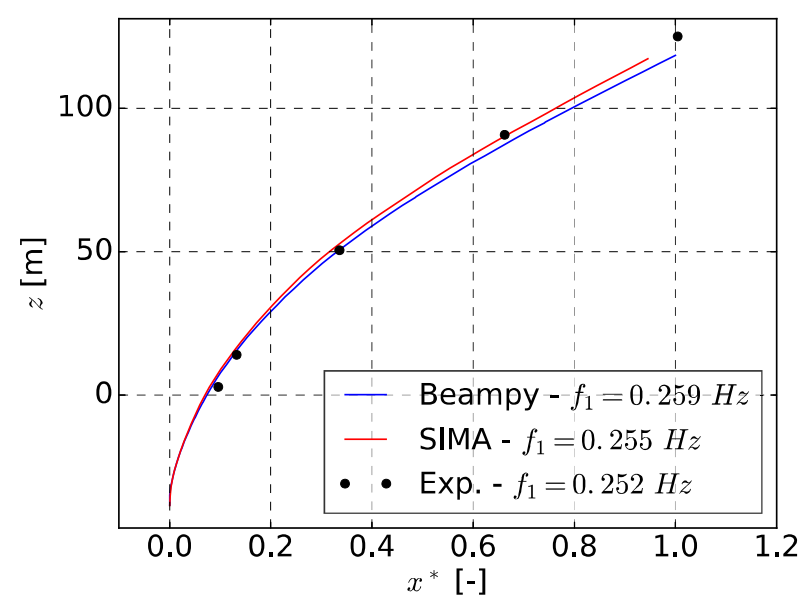

(a) Mode 1

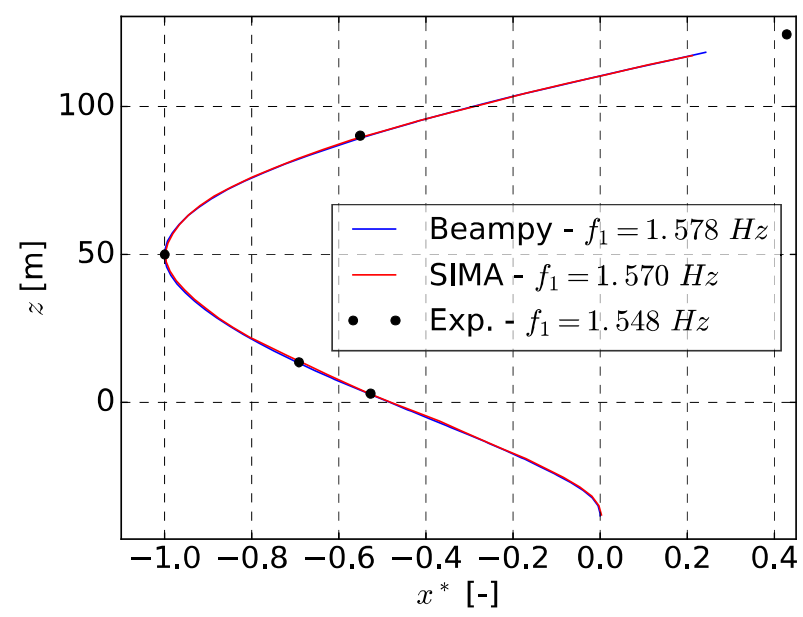

(b) Mode 2

Figure 9: Comparison of the computed and measured fore-aft bending modes normalised shapes.

At $\mathrm{T}=10 \mathrm{~s}$ (Figure 11c), the $k R$ number is lower and the Morison based numerical model (SIMA) should be more appropriate to compute the hydrodynamic loads. However the non-linear potential flow model shows a very good agreement with the experiments and with SIMA.

The two first harmonics in the steady-state mudline bending moment series are plotted in Figure 12. The measurements are shown in grey, indicating as well their uncertainty (see Appendix B). When $T=6 \mathrm{~s}$ (Figure 12a), the agreement between WS_CN-FEM and the experiments is very good (below $4 \%$ relative difference), which is expected for the current values of $k R$ and $\mathrm{KC}$, except for the first harmonic of the case at $H / \lambda=1 / 22$ where the response is much larger in the experiments. Several factors may contribute to this underestimation of the response. For $T=6 \mathrm{~s}$, the diffraction due to the cylinder that is observed in the tank may result in the reflection of diffracted waves. The large difference between the measured wave with and without the model (w8 vs. w11 in Figure 11) suggests that reflections or diffraction may be more important for this case, leading to larger loads in the experimental measurements. Additionally, the underestimation of the WS_CN-FEM solver in that case may be the result of the Weak-Scatterer approximation, which assumes a small perturbation on the incident flow (potential and free-surface elevation). Particularly, the Weak-Scatterer approach has been shown to poorly represent the run-up on a free-surface piercing cylinder [6].

For the other cases, $T=8 \mathrm{~s}$ and $10 \mathrm{~s}$, the agreement is good on the first harmonic, with a relative difference between WS_CN-FEM and the experiments below 2\%. Figure $12 \mathrm{~b}$ also shows that when the first fore-aft bending mode is excited (at $\mathrm{T}=8 \mathrm{~s}$ ), SIMA tends to over-estimate the amplitude of the $2^{\text {nd }}$ harmonic, which is consistent with expectations [14, 18], and the potential flow solver WS_CN-FEM seems to underestimates it. The double frequency harmonic is almost $30 \%$ smaller in WS_CN-FEM than in the experiments for the steepest case at $T=8 \mathrm{~s}$, and SIMA overestimates it by $24 \%$. In that case, the KC number can be too high and the $k R$ number too low for a valid applicability of the potential flow theory (see Figure 7). The uncertainty of the measurements is also much larger at the resonance because of the large elastic effects and the strong dependence of the response on the damping.

At $T=10$ s (Figure $12 \mathrm{c}$ ), the $2^{\text {nd }}$ bending moment harmonic is slightly overestimated by the two numerical models. WS_CN-FEM and SIMA overestimate the double frequency harmonic by $15 \%$ and $7 \%$, respectively. The relative difference seems to decrease when the wave steepness increases, hence the numerical models should be compared with experiments in steeper waves to better understand the reasons for these differences. The non-linear potential flow solver seems to correctly capture the hydroelastic effects at this wave period, despite the large $\mathrm{KC}$ number and the viscous effects that might occur. When the waves become large on the experimental model, the viscous effects may be substantial and would be overestimated because of scale effects: the scale factor is $1 / 50$ and the Reynolds number on the model is lower than at full scale when using Froude similarity.

The response at higher order harmonics is also studied with the non-linear coupling WS_CN-FEM, while the SIMA simulations employ a $2^{\text {nd }}$ order Stokes wave model. $3^{\text {rd }}$ order effects are however visible in the bending moment from SIMA as it is calculated by integration of the wave loads on the wetted surface up to the $2^{\text {nd }}$ order free-surface elevation. The $3^{\text {rd }}$ order bending moment computed by SIMA is hence only partial. The triple-frequency harmonics are plotted for all cases in Figure 13. One can see that the calculation of $W S \_C N-F E M$ is close to the experiments. The maximum steepness of the studied waves is not very large $(H / \lambda=1 / 22)$, hence the $3^{\text {rd }}$ order loads are small and the resulting $3^{\text {rd }}$ bending moment harmonic is only $2 \%$ of the $1^{\text {st }}$ bending moment harmonic for the case $T=10 \mathrm{~s}$. Despite the small amplitude, WS_CN-FEM is in good agreement with the experimental measurements. For instance, when $T=10 \mathrm{~s}$ and $H / \lambda=1 / 22$, it underestimates the bending moment by $30 \%$ but it is very near the measurement uncertainty zone. 


\section{Conclusion}

In this paper, a new hydroelastic model is studied for application to the modelling of large offshore structures. It includes a nonlinear hydrodynamic potential flow solver based on the Weak-Scatterer approach, tightly coupled to a structural model based on the Euler-Bernoulli beam theory. The weakly non-linear hydrodynamic solver computes the hydrodynamic loads, including the influence of the elastic response of the structure. This coupling is explicitly written in a coupled system of equations and solved in time-domain using a 4th order Runge-Kutta integrator. For now, only regular waves can be included in the hydrodynamic analysis. A fully nonlinear wave model is employed, based on the Rienecker and Fenton theory [24].

This hydroelastic model is applied to the case of a large diameter monopile, supporting an offshore wind turbine. The simulations results are compared with

1. experimental measurements made on a flexible $1 / 50$ Froude-scaled model, which includes the wind turbine tower and an RNA mass and damper and which respects Froude-scaled modal frequencies;

2. simulations results from SIMA, which here uses a Morison-based hydrodynamic model with $2^{\text {nd }}$ order wave kinematics, and neglects the viscous drag effects.

The load cases include a series of regular waves, with various steepnesses and periods. In particular, relatively small waves periods are considered $(6,8$ and $10 \mathrm{~s})$, in order to respect the applicability of the potential flow theory for sufficiently large $k R$ numbers. The observed agreement is good, except for the steepest wave at $T=6 \mathrm{~s}$, for which the measurements were perturbed by strong reflection on the wave tank walls. At $8 \mathrm{~s}$ wave period, the $2^{\text {nd }}$ order wave loads excite the first bending mode of the monopile, resulting in large oscillations at double frequency. While WS_CN-FEM underestimates their amplitude, SIMA overestimates it. The nonlinear potential flow method has also been shown to be accurate on the third harmonics, but steeper waves, with larger high-order effects should still be investigated. For now, the applicability of this potential flow-based hydroelastic model should be limited to relatively small KC numbers and large $k R$ numbers, where the diffraction remains dominant compared to viscous effects in the flow, but the agreement with the experiments at $T=10 \mathrm{~s}$ is very satisfactory, even in the steepest case where the $\mathrm{KC}$ number is large and where viscous effects should be non negligible. Application to larger wave periods should still, however, be investigated.

This method is not proven to be more efficient than a Morison-based hydroelastic solver for this type of application (analysis of a monopile), but it is a first validation step of the model before the extension of the modelling capability to the hydroelastic response of floating wind turbine platforms, for which the non-linear loads could play an important role. It should then account for large rigid-body motions of the platforms, in addition to the hydroelastic effects.

\section{Acknowledgements}

This work was carried out within the framework of the WEAMEC, West Atlantic Marine Energy Community, and with funding from the Pays de la Loire Region (France).

The authors thank Sintef Ocean and the other contributors of the WAS-XL (Research Council of Norway project 268182) project for sharing the experimental data used in this paper.

\section{References}

[1] Bachynski, E.E., Kristiansen, T., Thys, M., 2017. Experimental and numerical investigations of monopile ringing in irregular finite-depth water waves. Applied Ocean Research 68, 154-170. doi:dx. doi .org/ 10.1016/j. apor. 2017.08.011.

[2] Bachynski, E.E., Thys, M., Dadmarzi, F.H., 2020. Observations from hydrodynamic testing of a flexible, large-diameter monopile in irregular waves. Journal of Physics: Conference Series 1669, 012028. doi:10. 1088/1742-6596/1669/1/012028.

[3] Bak, C., Zahle, F., Bitsche, R., Taeseong, K., Yde, A., Henriksen, L.C., Hansen, M.H., Blasques, J.P.A.A., Gaunaa, M., Natajaran, A., 2013. The dtu 10-mw reference wind turbine.

[4] Borg, M., Hansen, A.M., Bredmose, H., 2016. Floating substructure flexibility of large volume $10 \mathrm{mw}$ offshore wind turbine platforms in dynamic calculations. Journal of Physics: Conference Series 753, 082024. doi:10.1088/1742-6596/753/8/082024.

[5] Chakrabarti, S.K., 1987. Hydrodynamics of offshore structures. WIT press.

[6] Chauvigné, C., 2016. Tenue à la mer d'un flotteur animé de grands mouvements pour les Energies Marines Renouvelables. Ph.D. thesis. Centrale Nantes.

[7] DNV GL AS, 2018. Support structures for wind turbines

[8] Ducrozet, G., Engsig-Karup, A.P., Bingham, H.B., Ferrant, P., 2014. A non-linear wave decomposition model for efficient wave-structure interaction. part a: Formulation, validations and analysis. Journal of Computational Physics 257, 863-883. doi:10.1016/j. jcp. 2013.09. 017.

[9] Faltinsen, O.M., 1990. Sea loads on ships and offshore structures. Cambridge University Press, Cambridge.

[10] Faltinsen, O.M., Newman, J.N., Vinje, T., 1995. Nonlinear wave loads on a slender vertical cylinder. Journal of Fluid Mechanics 289, 179198. doi:10.1017/S0022112095001297.

[11] Ferrant, P., 1998. Fully non-linear interactions of long-crested wave packets with a three-dimensional body, in: 22nd ONR Symposium on Naval Hydrodynamics, in Washington, D.C.

[12] Ferrant, P., Le Touzé, D., Pelletier, K., 2003. Non-linear time-domain models for irregular wave diraction about offshore structures. International journal for numerical methods in fluids 43, 1257-1277.

[13] Guignier, L., Courbois, A., Mariani, R., Choisnet, T., 2016. Multibody modelling of floating offshore wind turbine foundation for global loads analysis, in: Proceedings of the 26th International Ocean and Polar Engineering Conference, Rhodes, Greece, June 26-July 1.

[14] Horn, J.T.H., Krokstad, J.R., Amdahl, J., 2016. Hydro-elastic contributions to fatigue damage on a large monopile. Energy Procedia 94, 102-114. doi:10.1016/j.egypro.2016.09.203.

[15] Kim, J.H., Kim, Y., 2014. Numerical analysis on springing and whipping using fully-coupled fsi models. Ocean Engineering 91, 28-50. doi:10.1016/j. oceaneng. 2014.08.001.

[16] Kristiansen, T., Bachynski, E.E., Bickert, F., Hniche, A., Kocher, V., Liandrat, A., 2017. Aspects in model testing of a monopile in steep waves, in: Proceedings of the ASME 2017 36th International Conference on Ocean, Offshore and Arctic Engineering OMAE2017, p. V001T01A051.

[17] Kristiansen, T., Faltinsen, O.M., 2017. Higher harmonic wave loads on a vertical cylinder in finite water depth. Journal of Fluid Mechanics 833, 773-805. doi:10.1017/jfm.2017.702. 
[18] Krokstad, J.R., Stanberg, C.T., Nestgard, A., Marthinsen, T., 1998. A new nonslender ringing load approach verified against experiments. Journal of Offshore Mechanics and Arctic Engineering 120. doi:10. 1115/1.2829515.

[19] Lee, I.J., Kim, E.S., Kwon, S.H., 2020. A 3d direct coupling method for steady ship hydroelastic analysis. Journal of Fluids and Structures 94, 102891. doi:10.1016/j. jfluidstructs. 2020.102891.

[20] Letournel, L., 2014. Développement d'un outil de simulation numérique basé sur l'approche "Weak-Scatterer" pour l'étude des systèmes houlomoteurs en grands mouvements. Ph.D. thesis. Centrale Nantes. URL: https://hal archives-ouvertes.fr/tel-01207477.

[21] Letournel, L., Chauvigné, C., Gelly, B., Babarit, A., Ducrozet, G., Ferrant, P., 2018. Weakly nonlinear modeling of submerged wave energy converters. Applied Ocean Research 75, 201-222. doi:10. 1016/j.apor. 2018.03.014.

[22] Luan, C., Gao, Z., Moan, T., 2017. Development and verification of a time-domain approach for determining forces and moments in structural components of floaters with an application to floating wind turbines. Marine Structures 51, 87-109. doi:10.1016/j.marstruc. 2016. 10.002.

[23] MacCamy, R.C., Fuchs, R.A., 1954. Wave forces on piles: A diffraction theory. No. TM-69. Technical Report. Corps of engineers Washington DC beach erosion board.

[24] Rienecker, M.M., Fenton, J.D., 1981. A fourier approximation method for steady water waves. Journal of Fluid Mechanics 104, 119 137. doi:10.1017/S0022112081002851.

[25] Robertson, A., Jonkman, J., Vorpahl, F., Popko, W., Qvist, J., Frøyd, L., Chen, X., Azcona, J., Uzunoglu, E., Guedes Soares, C., Luan, C., Yutong, H., Pengcheng, F., Yde, A., Larsen, T., Nichols, J., Buils, R., Lei, L., Anders Nygard, T., Manolas, D., Heege, A., Ringdalen Vatne, S., Ormberg, H., Duarte, T., Godreau, C., Fabricius Hansen, H., Wedel Nielsen, A., Riber, H., C., L.C., Abele, R., Beyer, F., Yamaguchi, A., K., J.J., Shin, H., Shi, W., Park, H., Alves, M., Guérinel, M., 2014. Offshore code comparison collaboration continuation within IEA wind task 30: Phase II results regarding a floating semisubmersible wind system (No. NREL/CP-5000-61154). Technical Report. National Renewable Energy Lab.(NREL), Golden, CO (United States).

[26] Schløer, S., Bredmose, H., Bingham, H.B., 2016. The influence of fully nonlinear wave forces on aero-hydro-elastic calculations of monopile wind turbines. Marine Structures 50, 162-188. doi:10. 1016/j. marstruc. 2016.06.004.

[27] SINTEF, a. Sima. URL: https://www.sintef.no/en/software/sima/. accessed: 2021-04-19.

[28] SINTEF, b. Was xl project. URL: https://www. sintef. no/projectweb/ was-x1/. accessed: 2021-04-19.

[29] Suja-Thauvin, L., Bachynski, E.E., Pierella, F., Borg, M., Krokstad, J.R., Bredmose, H., . Critical assessment of hydrodynamic load models for a monopile structure in finite water depth. Marine Structures 72, 102743. URL: https://www. sciencedirect. com/science/article/pii/S095183392030037X, doi:https://doi.org/10. 1016/j. marstruc. 2020.102743.

[30] Velarde, J., Bachynski, E.E., 2017. Design and fatigue analysis of monopile foundations to support the DTU $10 \mathrm{MW}$ offshore wind turbine. Energy Procedia 137, 3-13.

[31] Wuillaume, P.Y., 2019. Simulation numérique des opérations d'installation pour les fermes d'éoliennes offshore. Ph.D. thesis. Centrale Nantes.

[32] Xu, K., Zhang, M., Shao, Y., Gao, Z., Moan, T., 2019. Effect of wave nonlinearity on fatigue damage and extreme responses of a semi-submersible floating wind turbine. Applied Ocean Research 91, 101879. doi:10.1016/j.apor. 2019.101879.

[33] Zhang, Y., Teng, B., 2021. A nonlinear potential flow model for higher-harmonic wave loads and ringing response of a monopile. Ocean Engineering 222, 108574. doi:10.1016/j.oceaneng. 2021. 108574.

\section{A. Hybrid RK4 integration schemes}

For stability and accuracy reasons, the structural solver, which uses an RK4 scheme, needs a much smaller time step than that of the hydrodynamic solver. The time step of the latter needs to be reasonably large to limit the computational cost.

As explained in Section 2.3, the integration of the structural response has been embedded in the hydrodynamic integration. The coupled equations are solved together at each synchronous time-step, and the hydrodynamic force is then kept constant for a number of sub-steps in the structure response before the next synchronous hydro-elastic time-step.

To demonstrate the accuracy of this integration scheme, simulations have been made on the same system in mechanically forced oscillations. No water is here considered for simplicity reasons. A $500 \mathrm{kN}$ sinusoidal force is applied at a $5 \mathrm{~s}$ period at the RNA in the $\mathrm{x}$-direction. A ramp of $5 \mathrm{~s}$ is used at the start of the simulation. The reference case does not use any sub time-step and does a direct integration of the response of the structure using an implicit Newmark- $\beta$ integration with a time-step of $0.001 \mathrm{~s}$ (with $\gamma=0.5$ and $\beta=0.25$ ). The three other cases use an RK4 scheme using the same time-step of $0.001 \mathrm{~s}$ and 12 modes to compute the response of the structure and respectively update the force at the RNA every 10, 50, and 100 time steps.

The computed responses are plotted in Figure 14 and have a very satisfactory agreement.

\section{B. Uncertainty estimation in regular waves}

The error bars in Figs. 12 and 13 indicate the estimated uncertainty in the experimental results based on a combination of random uncertainty (assessed using 5 repetitions of the regular waves with steepness 1/30) and standard uncertainty. The contributions to standard uncertainty include the model dimensions ( $\pm 0.5 \mathrm{~mm}$ in the outer diameter, $\pm 0.1 \mathrm{~mm}$ for the inner core), mass distribution (10\% locally), water depth $( \pm 7 \mathrm{~mm}$ in model scale), wave elevation $( \pm 3 \%)$, damping level (ranging from $1 \%$ to $1.3 \%$ of critical for the first mode), and the measurement uncertainty in the strain gauges ( $0.4 \%$ on the total measurement).

Except for the measurement uncertainty in the strain gauges, other contributions to the standard uncertainty were propagated to the bending moment components numerically using the SIMA model, following a similar procedure that used by Bachynski et al. [1]. The wave elevation is the largest contributor to the uncertainty in the first harmonic moment. For higher harmonics, the uncertainty in the strain gauges is also important. For resonant responses, the uncertainties in the mass and damping dominate. 
A WS theory-based model for the hydroelastic analysis of OWT substructures

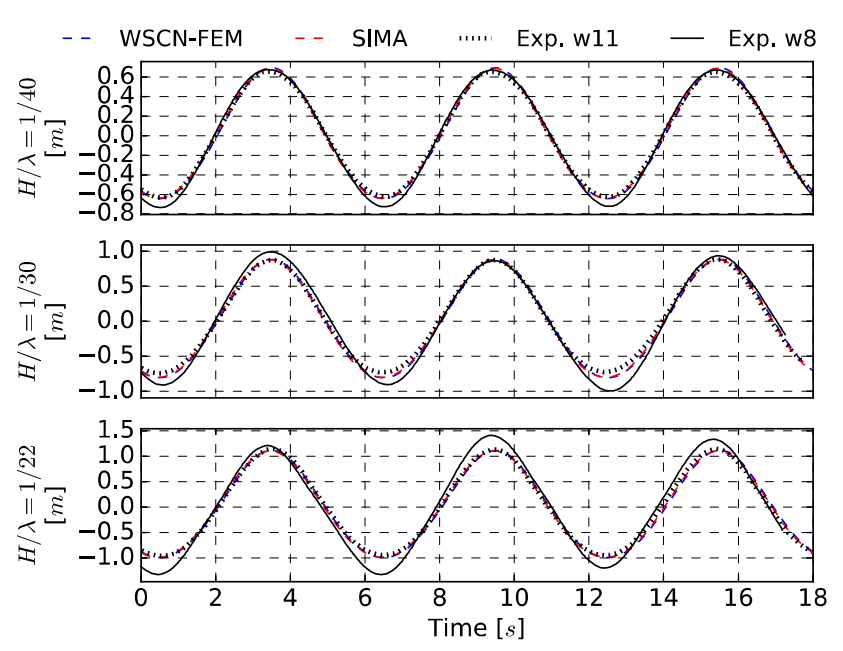

(a) $T=6 \mathrm{~s}$
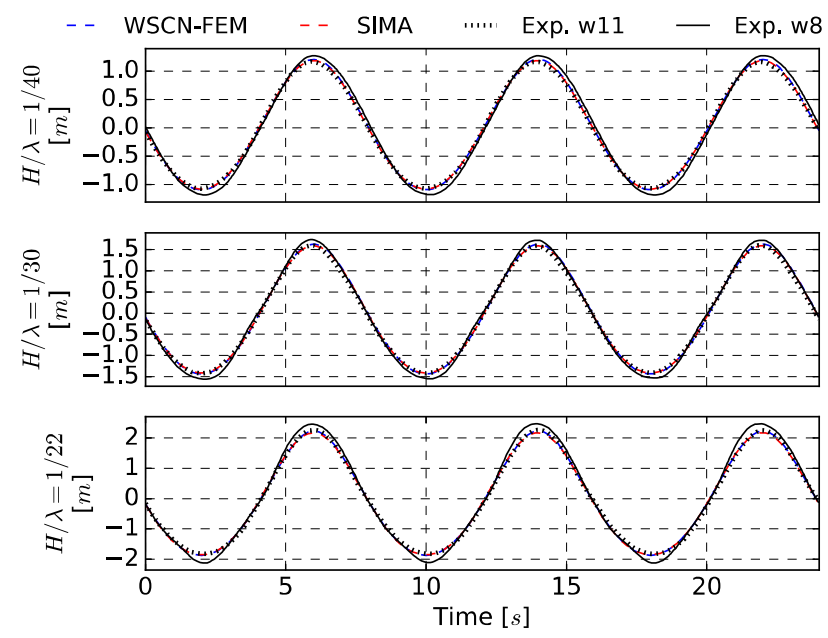

(b) $T=8 \mathrm{~s}$
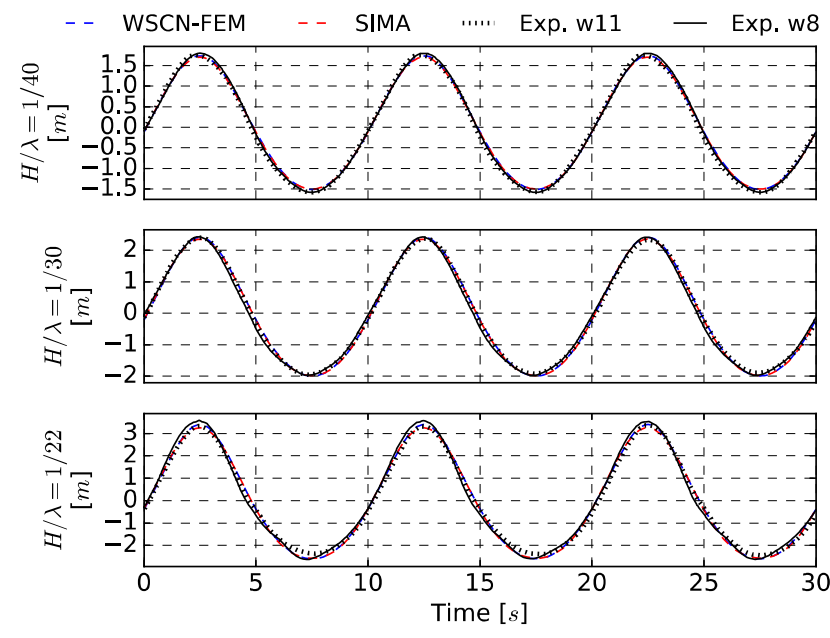

(c) $T=10 \mathrm{~s}$

Figure 10: Comparison of the computed and measured free surface elevation.


(a) $T=6 \mathrm{~s}$
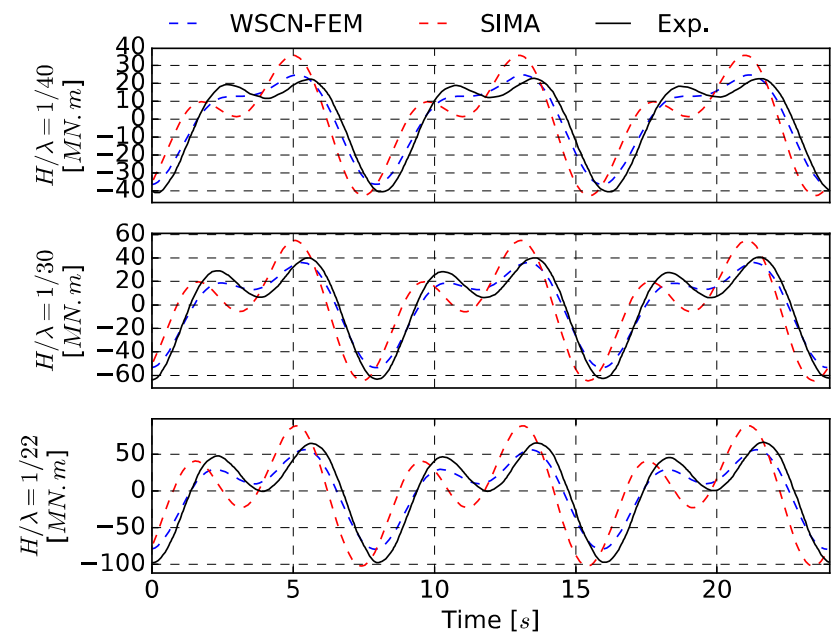

(b) $T=8 \mathrm{~s}$
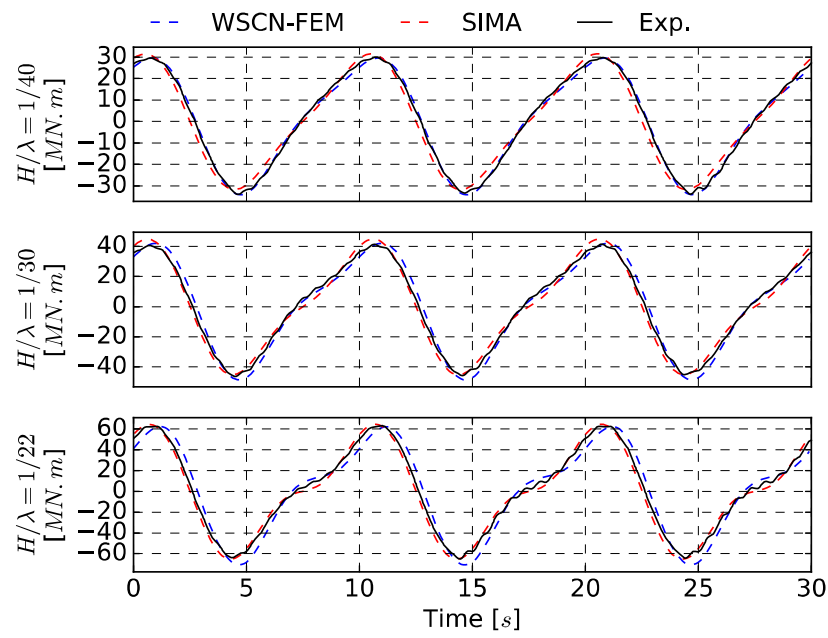

(c) $T=10 \mathrm{~s}$

Figure 11: Comparison of the computed and measured mudline bending moment time series. 

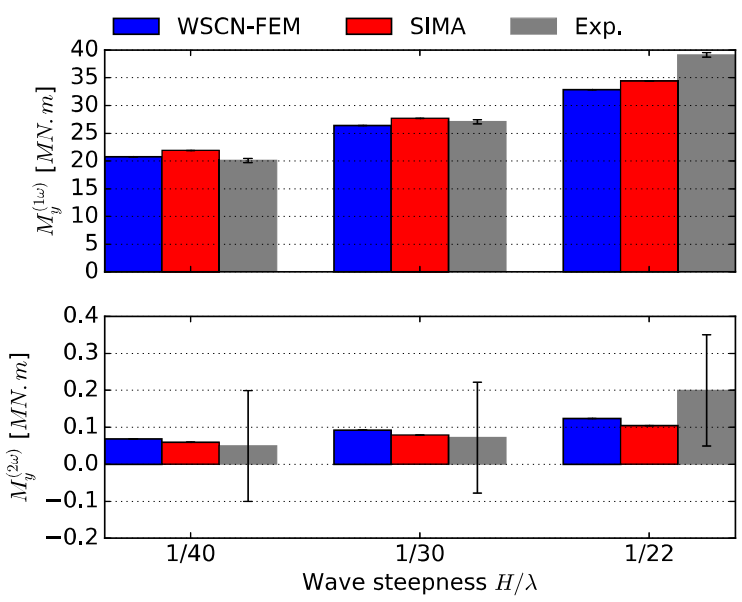

(a) $T=6 \mathrm{~s}$
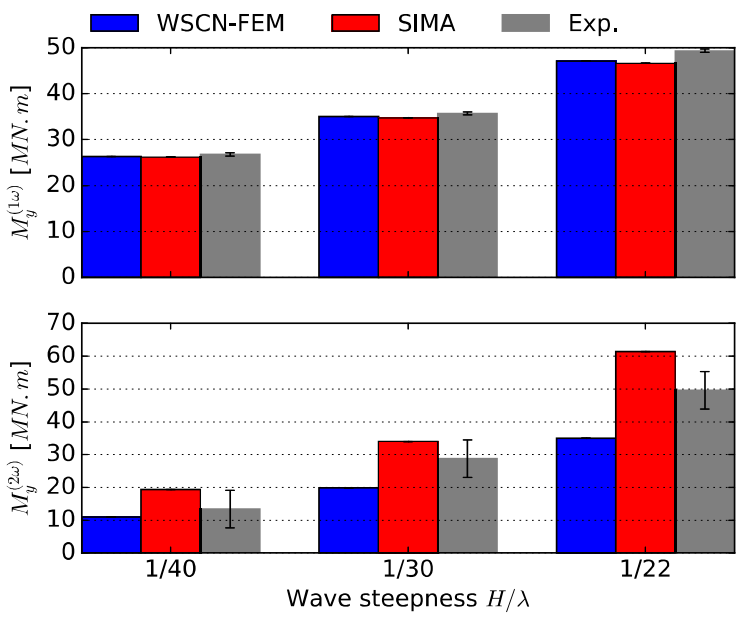

(b) $T=8 \mathrm{~s}$
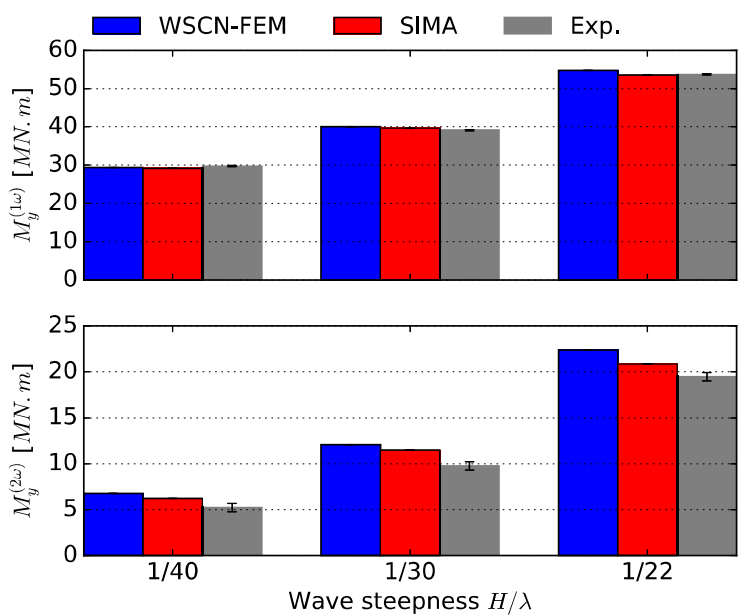

(c) $T=10 \mathrm{~s}$

Figure 12: Two first harmonics of the mudline bending moment, measured and simulated
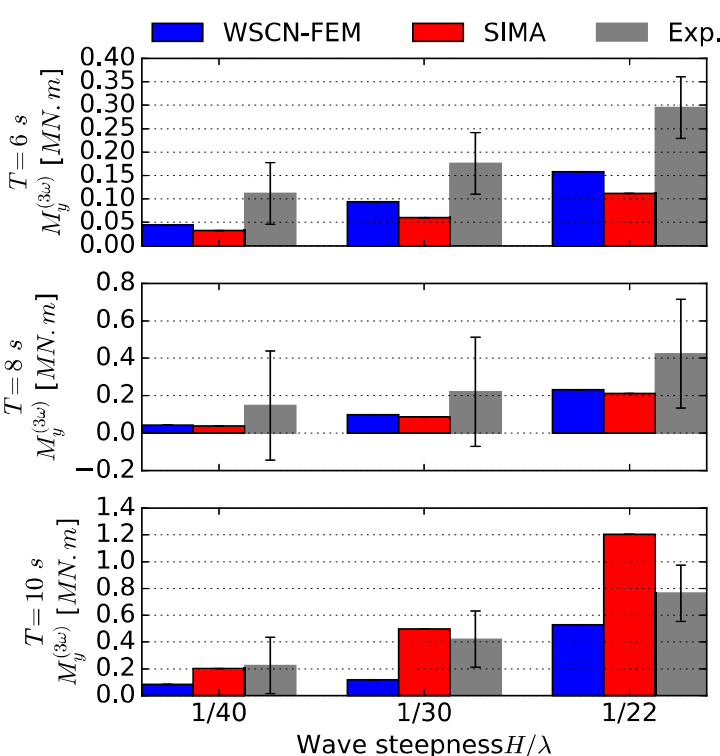

Figure 13: Third harmonics in all regular waves cases: $T=6 \mathrm{~s}$ (top), $6 \mathrm{~s}$ (middle) and $10 \mathrm{~s}$ (bottom) 
A WS theory-based model for the hydroelastic analysis of OWT substructures

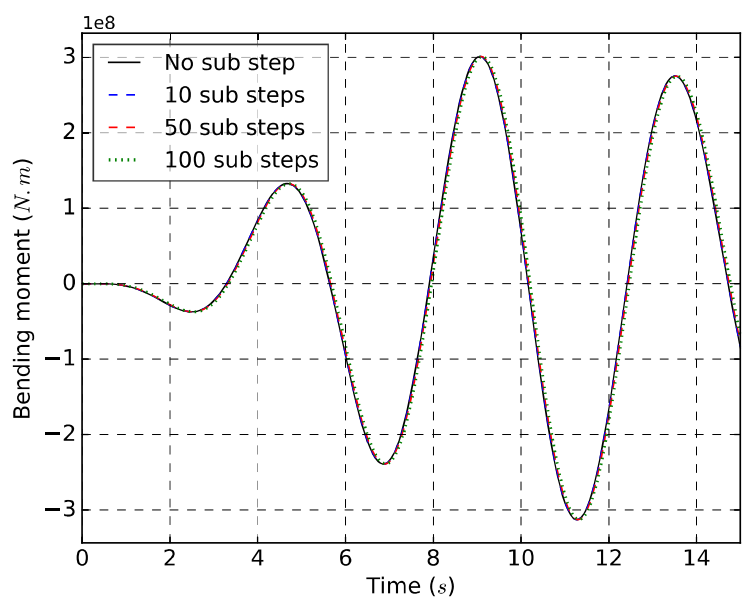

(a) Mudline bending moment

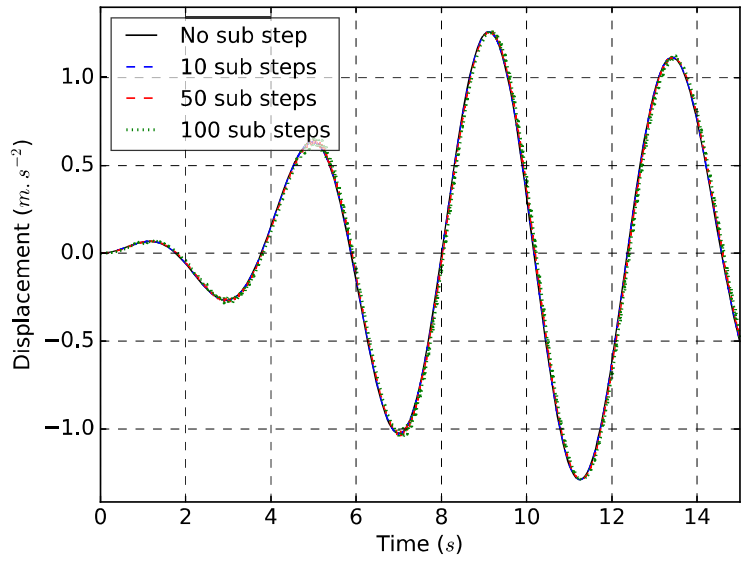

(b) Acceleration in the $\mathrm{x}$ direction at the RNA

Figure 14: Response of the structure in forced oscillations using different sub time-steps in the structural solver 